\title{
Surgical and endovascular treatment of secondary aortoesophageal fistula
}

Enrico Maria Marone, MD, ${ }^{\text {a }}$ Paolo Baccari, MD, ${ }^{\text {b }}$ Chiara Brioschi, MD, ${ }^{a}$ Yamume Tshomba, MD, ${ }^{\mathrm{a}}$ Carlo Staudacher, MD, and Roberto Chiesa, MD, ${ }^{a}$ Milan, Italy

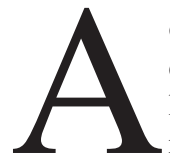

ortoesophageal fistula is an uncommon and highly fatal condition, even if opportunely treated. In rare cases it has been encountered in association with extensive corrosive injury. No cases of successful endovascular repair of aortoesophageal fistula caused by corrosives are reported in the English-language literature. We report a case of severe esophagogastritis caused by massive ingestion of hydrochloric acid complicated by aortoesophageal fistula. Combined surgical and endovascular treatment by means of a stent graft proved to be successful.

\section{Clinical Summary}

A 20-year-old man was brought to the emergency department after ingestion of hydrochloric acid with suicidal intent. At admission, he required endotracheal intubation, fluid resuscitation, and correction of metabolic acidosis. Endoscopy showed edema and necrosis of the palate, tongue, pharynx, larynx, epiglottis, and arytenoids. Computed tomography showed marked edematous thickening of the esophageal and gastric walls (Figure 1) and posterior gastric wall perforation with perigastric and perisplenic fluid effusion with free air. Urgent laparoscopy evidenced free blood in the supramesocolic area, necrosis, and perforation of the posterior wall of the stomach. The abdominal esophagus seemed spared. A laparoscopic total gastrectomy was completed. At the last phase of the operation, brisk arterial bleeding coming from the preaortic necrotic tissues ensued. After conversion in laparotomy, the hemostasis was optimized. A tube was then inserted in the esophageal stump, and a nutritional jejunostomy was accomplished.

Twenty-five days after the operation, sudden bleeding from the esophageal tube with hemorrhagic shock ensued. An aortoesophageal fistula from the supraceliac malacic aortic wall was recognized on emergency left thoracophrenolaparotomy. The thoracic aorta was clamped, and the breach was closed with direct sutures. This was the life-saving procedure of choice to stop the bleeding. The treatment was not considered definitive because the risk of new bleeding from the aorta seemed to be very high.

From the Departments of Vascular Surgery a and General Surgery, "VitaSalute" University, Scientific Institute H. San Raffaele, Milan, Italy.

Received for publication Nov 29, 2005; accepted for publication Jan 13, 2006.

Address for reprints: Enrico Maria Marone, MD, Chirurgia Vascolare, IRCCS H. San Raffaele, Via Olgettina 60, 20132 Milano, Italy (E-mail: marone.enrico@hsr.it).

J Thorac Cardiovasc Surg 2006;131:1409-10

$0022-5223 / \$ 32.00$

Copyright $\odot 2006$ by The American Association for Thoracic Surgery doi:10.1016/j.jtcvs.2006.01.036

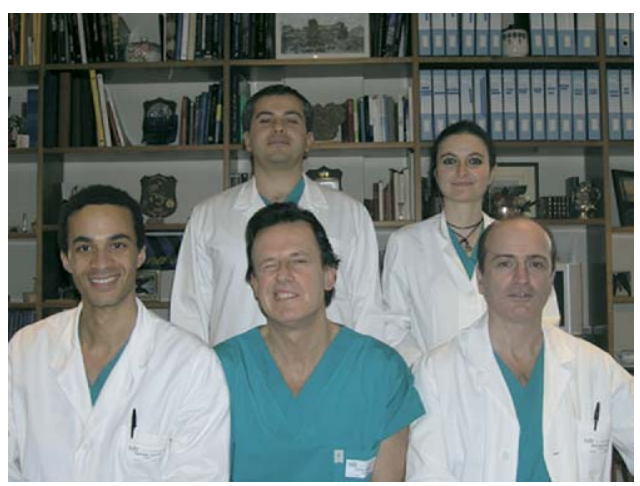

Drs Tshomba, Marone (standing), Chiesa (seated), Brioschi (standing), and Baccari (seated) (left to right).

aorta was

successfully

performed

the day after. The common femoral artery was exposed through a right groin incision. Through a left percutaneous femoral access, an angiogram localized the celiac axis, which was marked with a guide wire as the distal limit of the endograft. A 0.035-inch Amplatz super-stiff guide wire (Boston Scientific, Natick, Mass) was then passed up to the ascending aorta. The $18 \mathrm{~F}$ delivery sheath was advanced in the distal descending thoracic aorta. A straight $26 \times 238-\mathrm{mm}$ Zenith self-expanding endograft (William Cook Europe A/S, Bjaeverskov, Denmark) was positioned to terminate immediately above the celiac axis. The completion angiogram showed satisfactory endograft deployment. One month later, a computed tomographic scan showed correct positioning of the endoprosthesis (Figure 2). The esophagus developed strictures and was replaced by means of retrosternal coloplasty. At 1-year follow-up, the endoprosthesis was shown to be correctly positioned, with no signs of infection and with persistent exclusion of the fistula.

\section{Discussion}

Aortoesophageal fistula is an uncommon and highly fatal condition, occurring most commonly in association with thoracic aortic aneurysms or aortic prosthetic grafts, ruptured atherosclerotic ulcers in the thoracic aorta, foreign bodies, esophageal or bronchogenic malignancies, esophageal resections, esophageal stenting, penetrating peptic esophageal ulcers, and traumas. ${ }^{1}$ Only a few

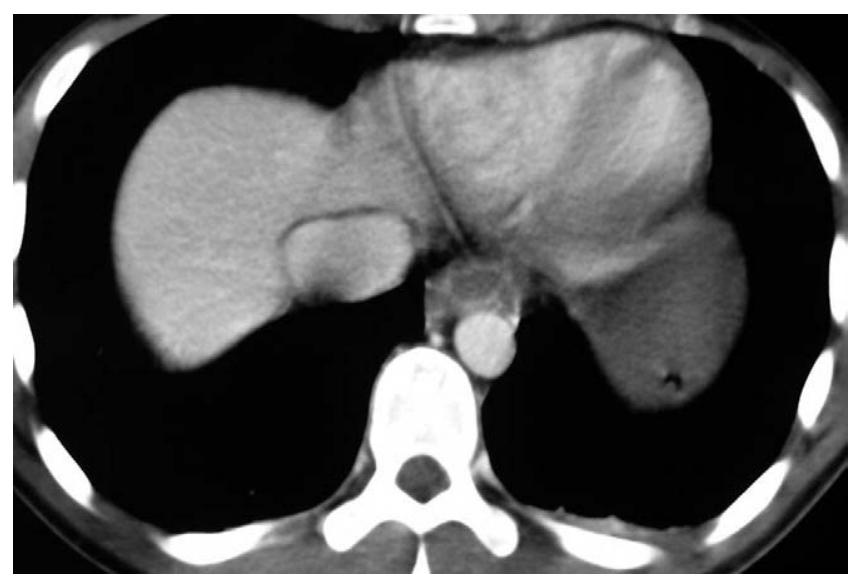

Figure 1. Preoperative computed tomographic scan showing marked edematous thickening of the esophageal wall. 


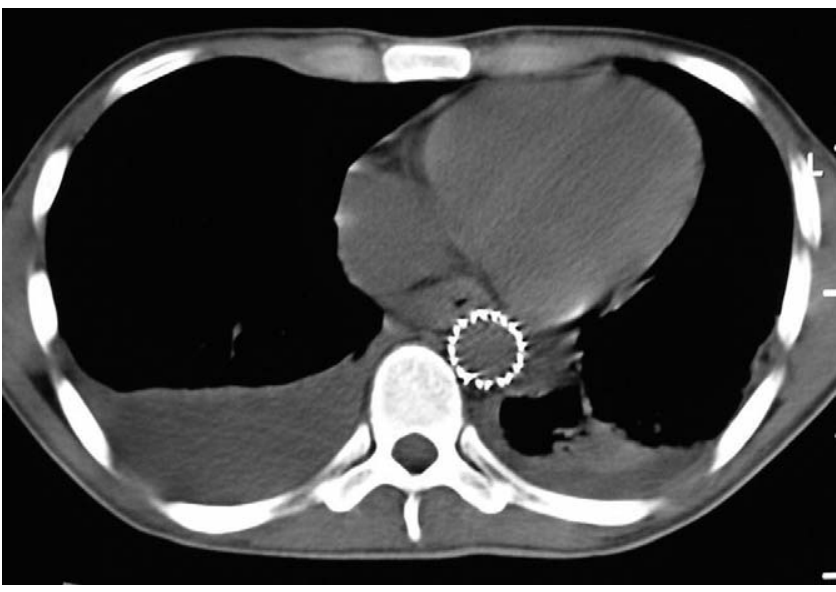

Figure 2. Postoperative computed tomographic scan showing correct positioning of the endoprosthesis and reduction in inflammation of mediastinal fat tissue.

cases of aortoesophageal fistula caused by corrosive ingestion are reported in the literature.

In cases of surgically repaired aortoesophageal fistulas reported in the literature, ${ }^{2,3}$ suture or prosthetic graft replacement of the aorta did not prove to be an adequate treatment because death from secondary hemorrhage often occurred because of late dehiscence and reopening of the aortic breach. The first case of aortoesophageal fistula repair with an endovascular stent graft was reported by Kato and associates ${ }^{4}$ in 2000. In this case the fistula was caused by irradiation of an esophageal cancer, and therefore intervention was palliative. In our case the aim was a definitive and minimally invasive treatment in a critically ill young man with impending coagulopathy. Endovascular treatment after primary repair of the laceration in the esophagus and in the aorta and after extensive debridement was the only way to definitively treat this condition while avoiding further surgical stress to the patient. In our case the infective risk for the endoprosthesis was minimal because of preoperative broad-spectrum antibiotic therapy and deployment of the graft inside an intact aortic wall. Successful surgical treatment of aortoesophageal fistulas caused by corrosive injury is rarely reported in the literature, and no cases of endovascular repair of this condition have been reported. Because surgical treatment alone is not able to result in a definitive solution, endovascular repair of the fistula and strengthening of malacic aortic walls by means of a stent graft can be a life-saving and successful approach. Further experience and follow-up will eventually confirm this result.

\section{References}

1. Maher MM, Murphy J, Dervan P, O'Connell D. Aorto-oesophageal fistula presenting as a submucosal oesophageal haematoma. $\mathrm{Br} J$ Radiol. 1998;71:972-4.

2. Lim CC, Cheah FK, Tan JC. Spiral computed tomography demonstration of aorto-oesophageal fistula from fish-bone. Clin Radiol. 2000;55: 976-7.

3. Reddi A, Chetty R. Primary aorto-esophageal fistula due to Takayasu's aortitis. Cardiovasc Pathol. 2003;12:112-4.

4. Kato N, Tadanori H, Tanaka K, Yasuda F, Iwata M, Kawarada Y, et al. Aortoesophageal fistula-relief of massive hematemesis with an endovascular stent-graft. Eur J Radiol. 2000;34:63-6.

\section{Surgical treatment of pulmonary artery sarcoma}

Neal C. Chhaya, MRCS, ${ }^{a}$ Andrew T. Goodwin, FRCS(CTh), ${ }^{b}$ David P Jenkins, FRCS(CTh), ${ }^{a}$ Joanna Pepke-Zaba, MD, and John J. Dunning, FRCS(CTh), ${ }^{a}$ Cambridge and Middlesbrough, United Kingdom

$\mathrm{P}$ rimary pulmonary artery angiosarcoma (PAS) is a rare, aggressive, and often fatal tumor of the main pulmonary artery that was first described by Mandelstamm ${ }^{1}$ in 1923. Only around 200 histologically confirmed incidences ${ }^{2}$ of PAS have been documented in the literature to date. The origin of

From Papworth Hospital, Papworth Everard, ${ }^{\mathrm{a}}$ Cambridge, and James Cook University Hospital, ${ }^{\mathrm{b}}$ Middlesbrough, United Kingdom.

Received for publication Oct 30, 2005; accepted for publication Nov 14, 2005.

Address for reprints: Mr Andrew Goodwin, Consultant Cardiothoracic Surgeon, James Cook University Hospital, Middlesbrough, TS4 3BW, United Kingdom (E-mail: andrew.goodwin@stees.nhs.uk).

J Thorac Cardiovasc Surg 2006;131:1410-1

$0022-5223 / \$ 32.00$

Copyright $\odot 2006$ by The American Association for Thoracic Surgery doi:10.1016/j.jtcvs.2005.11.045 these angiosarcomas is still uncertain, although characteristically they arise from pluripotent intimal cells possibly through the malignant development of intimal/subintimal myofibroblasts. ${ }^{3}$ These thoracic sarcomas generally arise from the pulmonary trunk, spreading to the main or proximal pulmonary arteries, with occasional involvement of the pulmonary valve. The diagnosis is clinically difficult to distinguish from chronic thromboembolic pulmonary hypertensive (CTEPH) disease owing to the similarity of symptoms at presentation. We describe our experience in a single institution.

\section{Clinical Summary}

Since 1988 we have treated 9 patients ( 5 female) with PAS whose ages ranging from 26 to 73 years (mean 44 years). Early in the series, 1 patient had a pneumonectomy and another had a heartlung transplant. Since 1999, of 250 patients undergoing pulmonary thromboendarterectomy (PTE), 6 have undergone attempted PTE 